\title{
Establishment of Specific Pathogen-Free Guinea-pig Colonies Using Limited-Flora Guinea-pigs Associated with Conventional Guinea-pig Flora, and Monitoring of their Cecal Flora
}

\author{
Makoto YANABE 1), Masaharu SHIBUYA ${ }^{1)}$, Toshihiko GONDA ${ }^{1)}$, Hidekazu ASAI 1), \\ Toshio TANAKA ${ }^{1)}$, Katuko SUDOU ${ }^{2)}$, Teruo NARITA ${ }^{3)}$, \\ Takane MATSUI4), and Kikuji ITOH${ }^{5)}$
}

\begin{abstract}
1)Japan SLC, Inc., 95-10 Aoi-cho, Hamamatsu, Shizuoka 433-8111, 2)Institute of Medical Science, The University of Tokyo, 4-6 Shiroganedai, Minato-ku, Tokyo 108-0071, ${ }^{3}$ SANKYO Co., Ltd., 1-2-58 Hiro-machi, Shinagawa-ku, Tokyo 140-0005, 4)Department of Veterinary Pathology, Obihiro University of Agriculture and Veterinary Medicine, Obihiro 080-8555, and 5) Laboratory of Veterinary Public Health, Graduate School of Agriculture and Life Sciences, The University of Tokyo, 1-1-1 Yayoi Bunkyo-ku, Tokyo 113-8657, Japan
\end{abstract}

\begin{abstract}
Six groups of limited flora (LF) Hartley guinea-pigs were produced by inoculation of hysterectomy-derived GF guinea-pigs with various combinations of cecal bacteria of conventional (CV) guinea-pigs to determine the effective bacterial cocktails for the establishment of a specific pathogen free (SPF) colony. Bifidobacterium magnum (Bif) isolated from CV guinea-pigs was used for pretreatment. The mortality of LF guinea-pigs inoculated with only Bif was $75 \%$, and that of those inoculated with Bif plus chloroform-treated cecal suspension (CHF) or Bif plus CHF plus 32 isolates from CV guinea-pigs was 40 to $66.7 \%$. These three groups were in an unhealthy condition with mucoid enteritis-like diarrhea. However, the mortality of LF guinea-pigs inoculated with the anaerobic growth on EG plates injected with $10^{-5}$ dilution of cecal contents (CF) or inoculated with Bif plus CF was 6.3 and $15 \%$, respectively. These latter two groups of LF guinea-pigs were transferred to separate barrier rooms and some of the LF guinea-pigs were maintained in isolators as a source of intestinal flora for SPF guinea-pigs. The composition of cecal flora of LF guinea-pigs was stable for a long time, and bacteroidaceae and peptococcaceae were maintained as predominant components. The basic composition of the cecal flora of SPF guinea-pigs originated from LF guinea-pigs, which consists mainly of the anaerobic bacteria, was not changed over a long period, and the flora composition became similar to that in CV guinea-pigs. Guinea-pig-specific pathogens from the SPF colonies were not detected during experiments.

Key words: cecal microflora, conventionalization, hysterectomy-derived animals, limited flora guinea-pigs, SPF guinea-pigs
\end{abstract}

(Received 5 April 2000 / Accepted 8 August 2000)

Address corresponding: K. Itoh, Laboratory of Veterinary Public Health, Graduate School of Agriculture and Life Sciences, The University of Tokyo, 1-1-1 Yayoi, Bunkyo-ku, Tokyo 113-8657, Japan 


\section{Introduction}

Specific pathogen-free (SPF) animals are generally produced using hysterectomy-derived animals maintained in barrier type animal facilities. However, the barrier system prevents not only the invasion of infectious agents but also normal floral organisms. Consequently, SPF animals lack the indigenous flora which resist the colonization of pathogenic species or opportunistic pathogens. Therefore, an artificial supply of indigenous flora is essential for hysterectomy-derived animals [2, 7].

Recently, standardization of the microflora of SPF laboratory animals has become necessary for several research fields. A few reports on the production of SPF guinea-pigs $[2,8]$ have been published and they reported that some SPF guinea-pig colonies suffered problems caused by poor intestinal flora. Control of the intestinal flora of SPF guinea-pigs is more important than that of SPF mice and rats because the ceca of germfree (GF) guinea-pigs become very large as they grow and ex-GF guinea-pigs are also very sensitive to enteric infections. Normalization of intestinal flora is essential at an early stage of life in SPF guinea-pigs.

In the present study, we attempted to establish breeding colonies of SPF guinea-pigs harboring normal intestinal flora using limited-flora (LF) guinea-pigs as a source of intestinal flora. We also monitored the cecal flora and pathogenic microbes of the SPF guineapigs and checked their fertility to confirm their normal status as a guinea-pig breeding colony.

\section{Materials and Methods}

Animals: Conventional (CV) Hartley guinea-pigs obtained from a commercial supplier (Funabashi Farm, Funabashi, Japan) were used as donors of cecal flora. Recipient GF guinea-pigs were derived by hysterectomy from Hartley guinea-pigs (Japan SLC, Inc., Hamamatsu, Japan) and raised under axenic conditions in flexible vinyl isolators.

Hand feeding of GF guinea-pigs: The GF guineapigs were reared in a hand rearing box set inside an isolator until 22 days of age and were subsequently transfered to an usual isolator. Heating was provided by means of heating pads controlled thermostatically. The guinea-pigs were resuscitated at $36^{\circ} \mathrm{C}$, and the temperature was maintained at $31^{\circ} \mathrm{C}$ until 5 days of age and then decreased stepwise to $25^{\circ} \mathrm{C}$ within five days. All animals were given $1 / 10$ volume $(\mathrm{m} / / \mathrm{g})$ of body weight of $50 \mathrm{kGy} \gamma$-irradiated milk (Commerciallyavailable milk; multiple vitamin mixture added at a rate of $2 \%$ ) by latex catheter twice daily until 33 days of age. From 34 to 40 days of age water was added to the milk and gradually increased. Autoclaved egg yolk was given until 32 days of age and $50 \mathrm{kGy} \gamma$-irradiated solid diet mixed with milk (Purina Guinea pig Chow 5025, USA) until 18 days of age ad libitum. The food was gradually changed to a solid diet.

Production of LF guinea-pigs: Bifidobacterium magnum (Bif) for pretreatment was isolated from $\mathrm{CV}$ guinea-pigs at 2 or 3 weeks of age. This species is specific and is the dominant lactic acid producing bacterium in the intestine of guinea-pigs. Cecal flora of $\mathrm{CV}$ guinea-pigs aged 10 weeks was used as the floral source. Culture methods were the same as those described previously [10]. A $10^{-5}$ dilution of cecal contents was inoculated into EG agar plates and incubated in an anaerobic chamber for 5 days (CF). Growth was suspended in anaerobic trypticase soy broth without dextrose (Difco, USA) and the mixture was inoculated into GF guinea-pigs. Chloroform-treated cecal suspension (CHF) [5] of CV guinea-pigs, and 32 strains consisting of Streptococcus spp., Bacillus spp., Bifidobacterium, Bacteroides spp. and Veillonella spp. isolated from ceca of CV guinea-pigs were used as the source of guinea-pig intestinal flora. Flora cultures were enclosed in a tube with a butyl-rubber stopper, transferred into isolators and inoculated into GF guineapigs between 12 and 21 days of age by injection of 0.5 $\mathrm{m} l$ of suspension into the stomach by means of a metal catheter attached to a syringe. Six groups of LF guineapigs were produced by a combination of four different inocula (Table 1).

Maintenance of LF guinea-pigs: Two groups of LF guinea-pigs were kept in large flexible vinyl isolators $(1150 \times 1200 \times 800 \mathrm{~mm})$ in the same way as GF animals, and given commercial diet (Purina Guinea-pig $5025)$ sterilized by $\gamma$-irradiation at $50 \mathrm{kGy}$ and autoclaved water ad libitum. Breeding was started using 6 males and 9 females in the Expt. 1 group, and 8 males and 9 females in the Expt. 2 group at 12 weeks of age.

Production and maintenance of SPF guinea-pigs: The Expt. 1 and 2 groups of LF guinea-pigs and their offspring were transferred from the isolators to barrier 
Table 1. Influence of oral inoculation with different microorganisms on the mortality of LF guinea-pigs

\begin{tabular}{ccclrr}
\hline & $\begin{array}{c}\text { Number of } \\
\text { animals }\end{array}$ & Pretreatment & \multicolumn{2}{c}{ Guinea-pig flora } & Mortality \\
\hline Expt. 1 & 16 & - & CF & $(14$ and 21*) & $6.3 \%$ \\
2 & 20 & Bif(12) & CF & $(14$ and 21) & $15.0 \%$ \\
3 & 15 & - & CHF + Bif & $(12$ and 19) & $40.0 \%$ \\
4 & 6 & Bif (12) & CHF & $(14$ and 21) & $66.7 \%$ \\
5 & 9 & Bif (12) & CHF + isolates (14 and 21) & $44.4 \%$ \\
6 & 4 & Bif(12) & \multicolumn{2}{c}{-} & $75.0 \%$ \\
\hline
\end{tabular}

CF: Growth of a $10^{-5}$ dilution of cecal contents of CV guinea-pigs on EG plates in an anaerobic chamber. Bif: Bifidobacteria magnaum isolated from cecal contents of CV guinea-pigs aged 2 or 3 weeks. CHF: Chloroform-treated cecal suspension. Isolates: 32 isolates from the ceca of CV guinea-pigs including Streptococcus spp. Bacillus spp. Bifidobacterium magnum, Bacteroides spp. and Veillonella spp. *Figures in parentheses are days after birth, when guinea-pigs were inoculated with Bif or flora.

rooms, 9 months and 3 years after association with cecal flora, respectively. Some of the LF guinea-pigs were maintained in the isolators to preserve their flora, and they were bred. The animals were kept in two barrier rooms at $25-27^{\circ} \mathrm{C}$ with a relative humidity of $45-65 \%$ under a lighting regimen of a $12 \mathrm{hr}$ light/dark cycle, and total air exchanges $12-15$ times/hr. They were housed in aluminium cages $(1200 \times 600 \times 420$ $\mathrm{mm}$ ), and fed commercial diet (5L08: PMI Feeds, Inc., USA) sterilized at $121^{\circ} \mathrm{C}$ for $20 \mathrm{~min}$. Acidified water (pH 2.5-3.0 adjusted with hydrochloric acid) was given ad libitum. Breeding was performed by a polygamous group system (1 male and 6 females, postpartum mating).

Culture of intestinal bacteria: Bacteriological procedures were the same as those described previously [10]. After serial 10-fold dilution of homogenized cecal contents in an anaerobic chamber, the diluted samples were spread on the surface of 11 selective and four nonselective media. Bacterial numbers were expressed as $\log _{10}$ counts of viable bacteria per gram wet weight of cecal content.

Histological examination: Several parts of the gastrointestinal tract were fixed with $10 \%$ buffered formalin and embedded in paraffin to prepare 4 to $5 \mu \mathrm{m} \mathrm{sec-}$ tions, which were stained with hematoxilyn-eosin.

Microbiologic monitoring: To determine the microbiological status in the SPF colonies, guinea-pigs were examined in the two breeding rooms at intervals of 1 month. Fresh feces, blood and tracheal swabs were tested for infections with Salmonella spp., Bordetella bronchiseptica, Pasteurella pneumotropica, Streptococcus zooepidemicus, Streptococcus pneumoniae, Pseudomonas aeruginosa, Tyzzer's organism, Mycoplasma spp., Sendai virus and Eimeria caviae by the methods described previously [11].

Fertility: For verification of fertility of the SPF guinea-pigs, the pregnancy rate, birth rate, litter size and weaning rate were examined.

This study was approved by the Laboratory Animal Use and Care Committee of the Faculty of Agriculture, the University of Tokyo.

\section{Results}

Production of LF guinea-pigs: Figure 1 shows the growth curve, and Table 1 the mortality rate of LF guinea-pigs. The mortality of LF guinea-pigs inoculated with only Bif was $75 \%$, and that of those inoculated with Bif plus CHF or Bif plus CHF plus 32 isolates was 40 or $66.7 \%$, respectively. The following clinical signs were observed in these groups: irregular weight gain, coarse fur, mucoid enteritis-like diarrhea and abdominal distention. Therefore, these groups (Expt. 3, 4, 5 and 6) were sacrificed by anesthesia with $\mathrm{CO}_{2}$ gas at 50 days of age. In these LF guinea-pigs, degeneration and desquamation of the epithelial cells, dilatation of the glandular cavity and small basophilic cell-like regenerative epithelium in the surface layer were observed in the lamina propria and epithelium of 

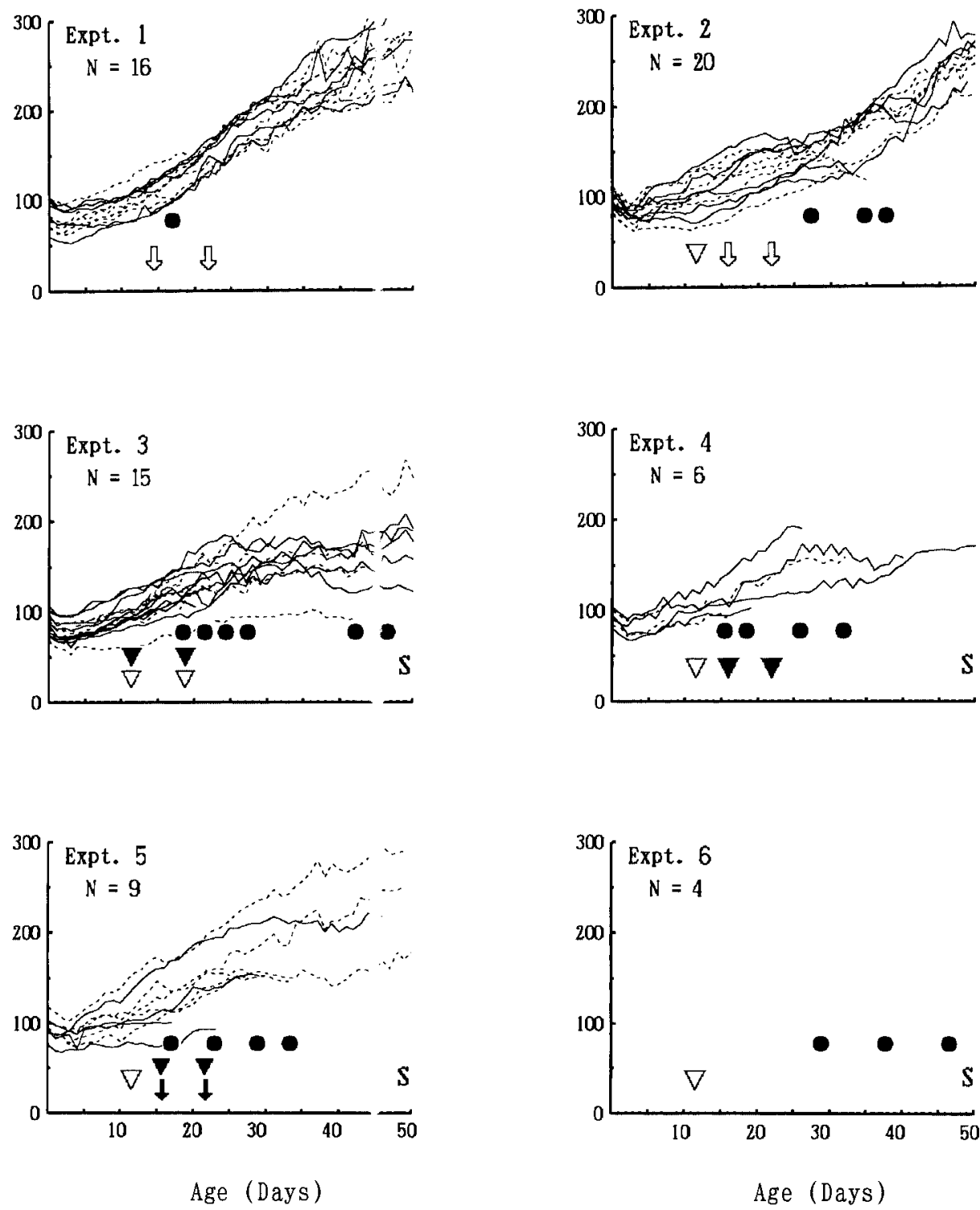

Fig. 1. Individual growth curve of limited-flora guinea-pigs. 9 , Death of individuals; $\nabla$, Inoculation of Bif;, Inoculation of CF; $\mathbf{\nabla}$, Inoculation of CHF; $\downarrow$, Inoculation of 32 strains (see the footnote of Table 1); S, Scrificed. No data of weight gain of guinea-pigs of Expt. 6.

the stomach (Fig. 2). Degeneration of epithelial cells, thickening of the mucosa and celluar infiltration of predominant neutrophils were seen in the lamina propria and epithelium of the cecum (Fig. 3). In the Expt. 1 and 2 groups, the mortality of ex-GF guinea-pigs inoculated with the CF or inoculated with Bif plus CF was 6.3 and $15 \%$, respectively. In the LF guinea-pigs of these two groups, no clinical signs were observed. The cause of death in these LF guinea-pigs appeared to be the mis-inoculation of milk into the respiratory tract.
The health condition of these groups was very good, and they were transferred into separate barrier rooms for the seed of SPF guinea-pigs. Some LF guinea-pigs were maintained in isolators to retain the basic intestinal flora of SPF guinea-pigs.

The composition of cecal flora of ex-GF and CV guinea-pigs used as donors of cecal flora are shown in Table 2. After 2 or 4 weeks, the cecal flora of the two groups of LF guinea-pigs was composed of bacteroidaceae and peptococcaceae as the predominant 


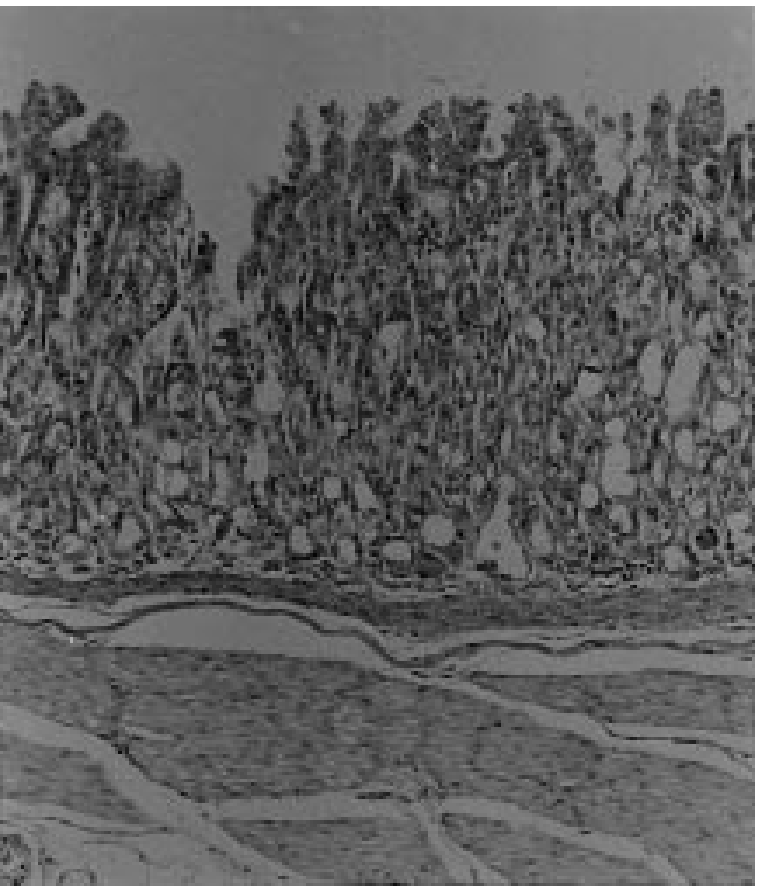

Fig. 2. Stomach of ex-GF guinea-pigs inoculated with $\mathrm{CHF}+\mathrm{Bif}$ in Expt. 3. Degeneration and desquamation of the epithelial cells, dilation of glandular. Cavity and small basophilic cell-like regenerative epithelium in the surface layer were observed.

flora, and clostridia, fusiform-shaped bacteria, bifidobacteria and eubacteria as the dominant flora. Among the aerobic bacteria, bacilli, corynebacteria, streptococci, staphylococci and enterobacteriaceae were detected in the Expt. 1 group. In the Expt. 2 group only streptococci were detected among aerobes. The major components of cecal flora of CV guinea-pigs, bacteroidaceae and peptococcaceae, were colonized in both groups of ex-GF guinea-pigs at the same level as in $\mathrm{CV}$ guinea-pigs.

Monitoring cecal flora of LF guinea-pigs: The results of the monitoring of cecal flora of the two groups of LF guinea-pigs reared and bred in isolators are shown in Table 2. Composition of their cecal flora was very stable for a long time. Bacteroidaceae and peptotococcaceae as the predominant population, clostridia and fusiform-shaped bacteria as the dominant population and aerobic bacteria as the low population were detected constantly. The numbers of bifidobacteria varied in both groups, and eubacteria were not detected in the Expt. 2 group.

SPF guinea-pigs: The changes of cecal flora of SPF

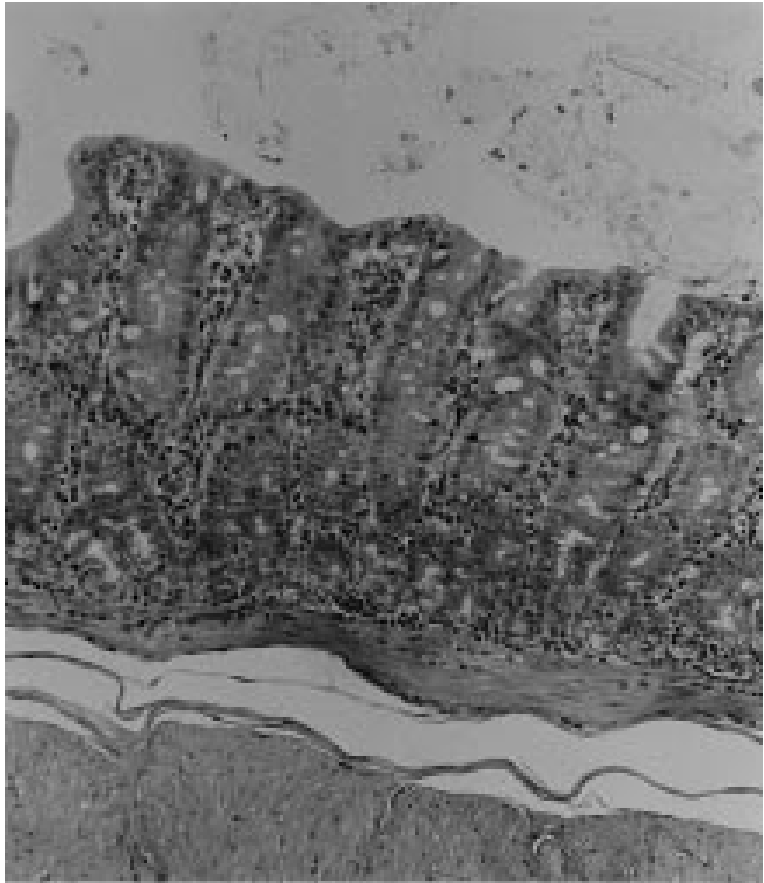

Fig. 3. Cecum of ex-GF guinea-pigs inoculated with $\mathrm{CHF}+\mathrm{Bif}$ in Expt. 3. Degeneration of epithelial cells, thickening of mucosa and cellular infiltration of predominant neutrophils were observed.

guinea-pigs are shown in Tables 3 and 4 . In both groups of SPF guinea-pigs, the changes of floral composition were similar. After transfer from each isolator to separate barrier-type rooms, lactobacilli and gemmiger, which had not colonized in the intestines of LF guineapigs, were detected in both groups. Thereafter, staphylococci and corynebacteria were undetectable. After transfer, the composition of cecal flora was stable in both colonies and became similar to that of $\mathrm{CV}$ guinea-pigs. The growth rate of SPF guinea-pigs was the same as that in Slc: Hartley guinea-pigs (Fig. 4).

Pathogens were not detected from the SPF guineapigs during the experimental period.

Fertility: Table 5 shows the fertility of the SPF guinea-pigs in colony A. The fertility of SPF guineapigs was as the same as that of Slc:Hartly guinea-pigs.

\section{Discussion}

Although the artificial supply of indigenous flora is essential for hysterectomy-derived animals to produce SPF animals $[2,5,10]$, only a few studies on the pro- 
Table 2. Cecal flora of LF guinea-pigs maintained in isolators after administration of cecal bacteria from conventional guinea-pigs

\begin{tabular}{|c|c|c|c|c|c|c|c|c|c|}
\hline \multirow{3}{*}{ Bacterial group } & \multirow{3}{*}{$\begin{array}{c}\mathrm{CV} \\
(\mathrm{N}=7)\end{array}$} & \multirow{2}{*}{\multicolumn{4}{|c|}{$\begin{array}{c}\text { Expt. } 1 \\
\text { Period after administration }\end{array}$}} & \multicolumn{4}{|c|}{ Expt.2 } \\
\hline & & & & & & \multicolumn{4}{|c|}{ Period after administration } \\
\hline & & $4 \mathrm{Ws}(\mathrm{N}=2)$ & $9 \mathrm{Ms}(\mathrm{N}=2)$ & $20 \mathrm{Ms}(\mathrm{N}=3)$ & $48 \mathrm{Ms}(\mathrm{N}=2)$ & $2 \mathrm{Ws}(\mathrm{N}=2)$ & $17 \mathrm{Ms}(\mathrm{N}=2)$ & $24 \mathrm{Ms}(\mathrm{N}=3)$ & $36 \mathrm{Ms}(\mathrm{N}=4)$ \\
\hline Bacteroidaceae & $9.1 \pm 0.1(7)$ & $10.0 \pm 0.1(2)^{*}$ & $9.7 \pm 0.1(2)^{*}$ & $9.4 \pm 0.2(3)$ & $9.5 \pm 0.6(2)$ & $10.2 \pm 0.2(2)$ & $10.1 \pm 0.2(2)$ & $10.4 \pm 0.2(3)$ & $10.4 \pm 0.3(4)$ \\
\hline Fusiform-shaped bacteria & $7.9 \pm 0.5(7)$ & $8.3 \quad(1)$ & $8.9 \pm 0.4(2)$ & $8.5 \quad(1)$ & & $8.8 \pm 0.0(2)$ & $8.0 \quad(1)$ & $8.2 \pm 0.5(2)$ & $8.9 \pm 0.2(2)$ \\
\hline Eubacteria & $7.6 \pm 1.4(5)$ & $8.1 \pm 1.0(2)$ & $8.0 \pm 0.0(2)$ & 9.6 & & $8.0 \pm 0.9(2)$ & & & \\
\hline Clostridia & $6.9 \pm 1.0(6)$ & $8.9 \pm 0.6(2)$ & $8.8 \pm 0.2(2)$ & & 8.5 & $9.1 \pm 0.3(2)$ & $9.2 \pm 0.0(2)$ & $9.0 \pm 0.6(3)$ & $9.6 \pm 0.3(4)$ \\
\hline Peptococaceae & $8.0 \pm 0.7(7)$ & $9.1 \pm 0.6(2)$ & $9.6 \pm 0.0(2)$ & $9.1 \pm 0.4(3)$ & $8.5 \pm 0.0(2)$ & $9.8 \pm 0.1(2)$ & $9.3 \pm 0.4(2)$ & $9.7 \pm 0.3(3)$ & $9.8 \pm 0.2(4)$ \\
\hline Bifidobacteria & $7.8 \pm 1.0(6)$ & $8.8 \pm 0.0(2)$ & $8.0 \pm 0.8(2)$ & $8.5 \pm 0.8(3)$ & $7.0 \pm 2.9(2)$ & $8.3 \pm 0.0(2)$ & $4.9 \pm 0.1(2)$ & $5.2 \pm 0.2(2)$ & $6.8 \pm 2.3(4)$ \\
\hline Veillonellaceae & $4.0 \pm 0.2(6)$ & $4.3 \pm 2.3(2)$ & $2.8 \pm 0.3(2)$ & $4.9 \quad(1)$ & $5.0 \quad(1)$ & $8.1 \pm 0.7(2)$ & $5.6 \pm 0.4(2)$ & $5.7 \pm 1.4(3)$ & $7.8 \pm 0.5(4)$ \\
\hline Gemmiger & & & & & & $8.5 \pm 0.2(2)$ & & & \\
\hline Lactobacilli & $5.3 \pm 1.6(3)$ & & & & & & & & \\
\hline Enterobacteriaceae & $2.9 \pm 0.1(2)$ & $6.8 \pm 0.6(2)$ & $6.7 \pm 1.4(2)$ & $8.3 \pm 0.9(3)$ & $7.1 \pm 0.8(2)$ & & $7.3 \pm 0.0$ & & \\
\hline Streptococci & $3.8 \pm 0.8(7)$ & $7.7 \pm 0.6(2)$ & $5.9 \pm 1.4(2)$ & $8.5 \pm 0.5(3)$ & $7.7 \pm 0.5(2)$ & $7.1 \pm 0.6(2)$ & & $8.8 \pm 0.5$ & $6.9 \pm 0.2(4)$ \\
\hline Staphylococci & & $5.9 \pm 1.3(2)$ & $3.3 \pm 0.0(2)$ & $7.3 \pm 0.4(3)$ & $5.5 \quad(1)$ & & & $7.7 \pm 0.4$ & $6.4 \pm 0.2(4)$ \\
\hline Pseudomonas aeruginosa & & & & & & & & & \\
\hline Corynebacteria & $4.3 \quad$ (1) & $7.1 \pm 0.0(2)$ & $4.9 \pm 0.1(2)$ & 7.7 & $7.7 \pm 0.5(2)$ & & & & \\
\hline Bacilli & $4.5 \pm 0.8(7)$ & $5.6 \quad(1)$ & $5.4 \pm 0.1(2)$ & & & & $7.6 \pm 0.1(2)$ & $7.8 \pm 0.4(3)$ & 6.3 \\
\hline Total counts & $9.4 \pm 0.4$ & $10.2 \pm 0.2$ & $10.0 \pm 0.0$ & $9.7 \pm 0.3$ & $9.2 \pm 0.3$ & $10.2 \pm 0.2$ & $10.2 \pm 0.2$ & $10.5 \pm 0.3$ & $10.6 \pm 0.2$ \\
\hline
\end{tabular}

* Mean \pm S.D. of log. counts of bacteria / $g$ feces when presents; figures in parentheses refer to the number of subjects that harbor the organisms.

Table 3. Change in cecal flora of SPF guinea-pigs in breeding colony A derived from LF guinea-pigs (Expt. 1)

\begin{tabular}{|c|c|c|c|c|c|}
\hline \multirow[b]{2}{*}{ Bacterial group } & \multirow{2}{*}{$\begin{array}{l}\text { Just transferred* } \\
\qquad(\mathrm{N}=2)\end{array}$} & \multicolumn{4}{|c|}{ Months after transfer } \\
\hline & & 3 months $(\mathrm{N}=7)$ & 2 years $(\mathrm{N}=3)$ & 3.5 years $(\mathrm{N}=5)$ & 5 years $(\mathrm{N}=5)$ \\
\hline Bacteroidaceae & $9.7 \pm 0.1(2)^{* *}$ & $9.5 \pm 0.3(7)$ & $9.3 \pm 0.1(3)$ & $9.2 \pm 0.2(5)$ & $9.6 \pm 0.1(5)$ \\
\hline Fusiform-shaped bacteria & $8.9 \pm 0.4(2)$ & $9.2 \pm 0.7(5)$ & $8.1 \pm 0.2(3)$ & $7.9 \pm 0.2(4)$ & $8.1 \pm 0.1(4)$ \\
\hline Eubacteria & $8.0 \pm 0.0(2)$ & $8.7 \pm 0.4(4)$ & $8.6 \pm 0.6(3)$ & $8.6 \pm 0.2(5)$ & $8.6 \pm 0.1(5)$ \\
\hline Clostridia & $8.8 \pm 0.2(2)$ & $6.8 \pm 2.5(7)$ & $8.5 \pm 0.6(3)$ & $8.5 \pm 0.1(5)$ & $8.8 \pm 0.2(2)$ \\
\hline Peptococcaceae & $9.6 \pm 0.0(2)$ & $9.3 \pm 0.3(7)$ & $9.3 \pm 0.2(3)$ & $9.2 \pm 0.3(5)$ & $9.8 \pm 0.1(5)$ \\
\hline Bifidobacteria & $8.0 \pm 0.8(2)$ & $8.6 \pm 0.2(4)$ & $8.3 \pm 0.4(2)$ & $8.6 \pm 0.2(5)$ & $7.9 \pm 1.4(5)$ \\
\hline Veillonellaceae & $2.8 \pm 0.3(2)$ & & $6.8 \pm 1.6(3)$ & $3.8 \pm 1.6(2)$ & $6.0 \pm 1.0(5)$ \\
\hline Gemmiger & & & $6.3 \pm 1.7(3)$ & $5.3 \pm 0.9(4)$ & $5.2 \pm 0.8(5)$ \\
\hline Lactobacilli & & & 5.0 & $3.9 \pm 0.2(2)$ & $3.9 \pm 0.3(3)$ \\
\hline Enterobacteriaceae & $6.7 \pm 1.4(2)$ & $4.3 \pm 2.3(5)$ & $5.6 \pm 1.4(2)$ & $3.4 \pm 0.1(3)$ & $3.9 \pm 0.2(5)$ \\
\hline Streptococci & $5.9 \pm 1.4(2)$ & $3.9 \pm 2.0(6)$ & $6.3 \pm 1.2(3)$ & & $5.9 \pm 0.7(5)$ \\
\hline Staphylococci & $3.3 \pm 0.0(2)$ & & $4.5 \pm 1.1(2)$ & & \\
\hline \multicolumn{6}{|l|}{ Pseudomonas aeruginosa } \\
\hline Corynebacteria & $6.6 \quad(1)$ & & & & \\
\hline Bacilli & $5.4 \pm 0.1(2)$ & $3.5 \pm 1.4(3)$ & $4.1 \pm 1.8(3)$ & & \\
\hline Total counts & $10.0 \pm 0.0$ & $10.0 \pm 0.2$ & $9.8 \pm 0.1$ & $9.6 \pm 0.2$ & $10.1 \pm 0.1$ \\
\hline
\end{tabular}

*See 9 months of Expt.1 in Table 7. **Means \pm S.D. of log. counts of bacteria / $\mathrm{g}$ feces when present; figures in parentheses refer to the number of subjects that harbor the organisms.

duction of SPF guinea-pigs have been reported $[2,8]$. In the present study we established SPF guinea-pig breeding colonies using LF guinea-pigs as artificial suppliers of indigenous flora, and monitored their cecal flora, pathogenic microbes and fertility in order to evaluate LF guinea-pigs as the source of intestinal flora for SPF guinea-pigs. Boot et al. [2] attempted to conventionalize GF guinea-pigs with host-specific cecal 
Table 4. Change in cecal flora of SPF guinea-pigs in breeding colony B derived from LF guinea-pigs (Expt. 2)

\begin{tabular}{|c|c|c|c|c|c|}
\hline \multirow[b]{2}{*}{ Bacterial group } & \multirow{2}{*}{$\begin{array}{l}\text { Just transferred* } \\
\qquad(\mathrm{N}=4)\end{array}$} & \multicolumn{4}{|c|}{ Months after transfer } \\
\hline & & 3 months $(\mathrm{N}=5)$ & 4 months $(\mathrm{N}=5)$ & 9 months $(\mathrm{N}=3)$ & 2 years $(\mathrm{N}=5)$ \\
\hline Bacteroidaceae & $10.4 \pm 0.3(4)^{* *}$ & $10.1 \pm 0.1(5)$ & $10.0 \pm 0.1(5)$ & $9.7 \pm 0.2(3)$ & $9.5 \pm 0.1(5)$ \\
\hline Fusiform-shaped bacteria & $8.9 \pm 0.2(2)$ & $8.7 \pm 0.0(2)$ & $8.2 \quad(1)$ & $8.0 \quad(1)$ & $8.5 \pm 0.3(2)$ \\
\hline Eubacteria & & & & $8.0 \quad(1)$ & $8.2 \pm 0.3(5)$ \\
\hline Clostridia & $9.6 \pm 0.3(4)$ & $9.4 \pm 0.3(5)$ & $9.3 \pm 0.4(5)$ & $8.8 \pm 0.4(3)$ & $8.9 \pm 0.2(5)$ \\
\hline Peptococcaceae & $9.8 \pm 0.2(4)$ & $9.1 \pm 0.3(5)$ & $10.0 \pm 0.1(5)$ & $9.9 \pm 0.0(3)$ & $9.8 \pm 0.2(5)$ \\
\hline Bifidobacteria & $6.8 \pm 2.3(4)$ & $7.6 \pm 2.1(5)$ & $8.3 \pm 1.3(5)$ & $8.2 \pm 0.4(3)$ & $9.2 \pm 0.3(5)$ \\
\hline Veillonellaceae & $7.8 \pm 0.5(4)$ & & $5.2 \pm 0.3(5)$ & $5.4 \pm 0.4(3)$ & $5.9 \pm 0.4(5)$ \\
\hline Gemmiger & & & $4.6 \pm 0.6(2)$ & $3.7 \pm 0.1(2)$ & $4.9 \pm 0.6(2)$ \\
\hline Lactobacilli & & & $4.0 \quad(1)$ & $5.1 \pm 0.9(3)$ & $4.0 \pm 0.3(2)$ \\
\hline Enterobacteriaceae & & $5.1 \pm 0.6(5)$ & $6.3 \pm 1.3(5)$ & $4.6 \pm 0.9(3)$ & $3.8 \pm 0.5(5)$ \\
\hline Streptococci & $6.9 \pm 0.2(4)$ & $6.2 \pm 0.4(5)$ & $4.8 \pm 0.8(5)$ & $5.9 \pm 0.5(3)$ & $3.6 \pm 0.3(5)$ \\
\hline Staphylococci & $6.4 \pm 0.2(2)$ & $6.4 \pm 0.4(5)$ & & & \\
\hline \multicolumn{6}{|l|}{ Pseudomonas aeruginosa } \\
\hline \multicolumn{6}{|l|}{ Corynebacteria } \\
\hline Bacilli & $6.3 \quad(1)$ & $5.8 \pm 1.0(4)$ & $4.3 \pm 1.1(4)$ & $3.9 \pm 0.2(2)$ & $4.1 \pm 0.3(5)$ \\
\hline Total counts & $10.6 \pm 0.2$ & $10.3 \pm 0.1$ & $10.3 \pm 0.1$ & $10.1 \pm 0.1$ & $10.1 \pm 0.1$ \\
\hline
\end{tabular}

*See 36 months of Expt. 2 in Table 7. **Mean \pm S.D. of log. counts of bacteria / g feces when present; figures in parentheses refer to the number of subjects that harbor the organisms.

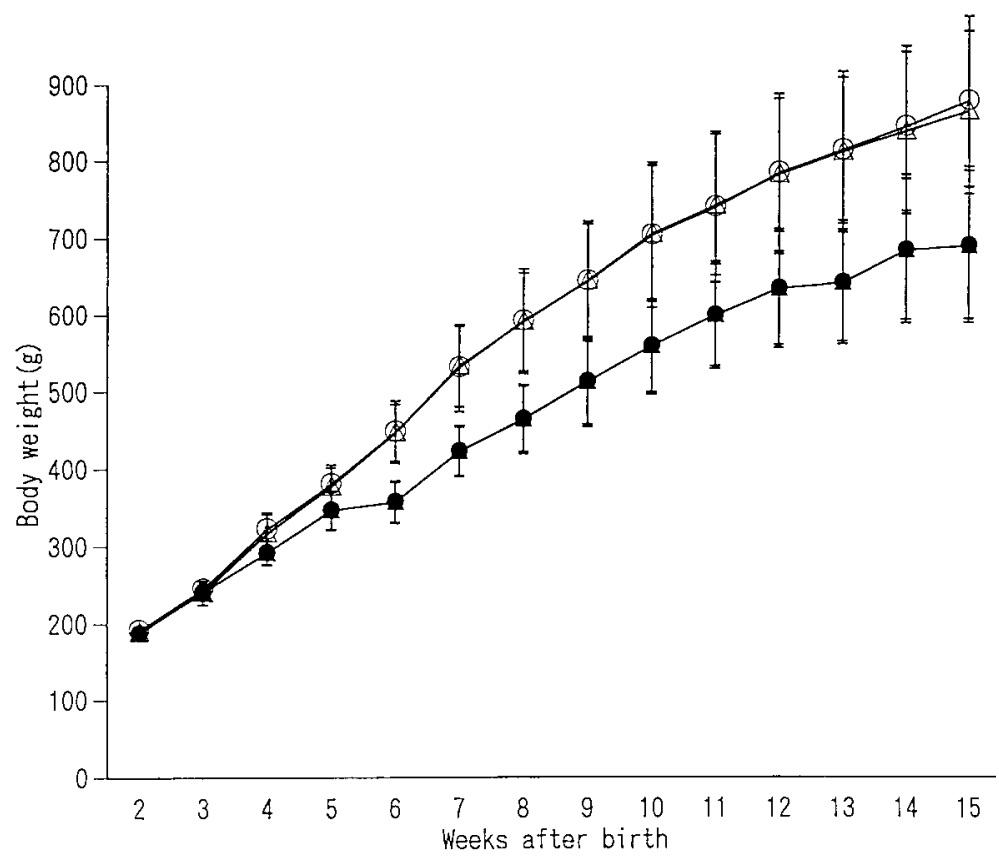

Fig. 4. Change of mean body weight in SPF guinea-pigs and Slc: Hartly. $(\mathrm{N}=10)$. OSPF male; $\mathbf{e S P F}$ male; $\triangle$ Slc: Hartly male; $\Delta$ Slc: Hartly female.

microflora. They concluded that colonization-resistant flora of mouse origin (mCRF) are not suitable for conventionalization of GF guinea-pigs. They reported opportunistic infections such as mastitis, cecitis, enteri- tis, otitis media and pneumonia with usually non-pathogenic bacterial species or groups [3], and Clostridium difficile-associated cecitis [1] in SPF guinea-pigs colonized with mCRF. These reports indicated that the 
Table 5. Fertility of SPF guinea-pigs derived for LF guinea-pigs

\begin{tabular}{ccccccc}
\hline $\begin{array}{c}\text { Breeding } \\
\text { colony }\end{array}$ & $\begin{array}{c}\text { No. of } \\
\text { female }\end{array}$ & $\begin{array}{c}\text { Rate of } \\
\text { pregnancy }\end{array}$ & $\begin{array}{c}\text { Birth } \\
\text { rate }\end{array}$ & $\begin{array}{c}\text { Litter size } \\
\text { mean } \pm \text { S.D. }\end{array}$ & $\begin{array}{c}\text { Weaned } \\
\text { mean } \pm \text { S.D. }\end{array}$ & $\begin{array}{c}\text { Weaning } \\
\text { rate }\end{array}$ \\
\hline A & 200 & $94.0 \% *$ & $94.7 \%^{*}$ & $3.9 \pm 1.3^{* *}$ & $3.2 \pm 1.2^{* *}$ & $82.0 \% * *$ \\
Cont. $^{+}$ & 70 & $91.4 \% *$ & $93.8 \%^{*}$ & $3.5 \pm 0.8^{*}$ & $3.0 \pm 0.7^{*}$ & $86.9 \% *$ \\
\hline
\end{tabular}

*Data of primipara. **Data of primipara to 6th parturition. ${ }^{\dagger}$ Slc: Hartly.

problems might be due to poor or unsuitable components of the intestinal flora. Moreover, Boot et al. [2] reported that almost complete conventionalization of GF guinea-pigs can be obtained by using a host or species specific enterobacteriaceae-free cecal flora produced by oral inoculation with trimetoplin. The use of this species specific flora will presumably reduce the rate of opportunistic infection, mainly enteric infections, in hysterectomy-derived barrier-maintained guinea-pigs.

In the Exp. 3 to 6 groups in which ex-GF guineapigs were not inoculated with $\mathrm{CF}$ (cultured whole flora), the mortality rate was very high, and there were no differences in histopathological lesions of the gastrointestinal tract among the four groups. These results indicate that GF guinea-pigs require contact with complex intestinal bacteria for conventionalization, and bifidobacteria, CHF, 32 isolates from CV guinea-pigs and mixtures of them may be insufficient as intestinal bacteria. Our results in this study of guinea-pigs and a previous study of rabbits [10] indicate that inoculation of cultured whole flora is a suitable method to convert GF animals to a normal state. Anaerobic culture of diluted cecal contents may select the effective anaerobes and eliminate the harmful microbes.

In SPF guinea-pigs, lactobacilli which could not be detected in the isolators were detected at low population levels, but the basic composition of cecal flora, consisting mainly of bacteroidaceae and peptococaceae, had not changed, and their flora finally became similar to that of CV guinea-pigs. Staphylococci and corynebacteria were eliminated after LF guinea-pigs were transferred to SPF colonies, and enterobacteriaceae, streptococci and bacilli were restricted at a low population level. These results indicated that bacteroidaceae and peptococcaceae are essential floral organisms in guinea-pigs.

In a previous study [10], we reported that pretreatment with Bacteroides before inoculation with whole flora of CV rabbits was indispensable to conventionalize GF rabbits. However, pretreatment with Bifidobacterium magnum was not necessary to conventionalize GF guinea-pigs as in the case of mice and rats. The intestinal flora of rabbits are different from those of other animals. In rabbits, no or very few lactic acid producing bacteria, bifidobacteria or lactobacilli, are detected in the intestines and small numbers of intestinal bacteria colonize the small intestine. This unique composition of intestinal bacteria may require pretreatment before GF rabbits are given whole flora.

In mice and rats, clostridia have been reported $[4,5]$ as the most important bacteria for conventionalization of GF animals and the colonization resistant factor [6, 9]. However, our data and the report published by Boot et al. [2] showed that clostridia in the intestines of guinea-pigs is unlikely to be key bacteria in control of the intestinal flora.

In the present study, LF guinea-pigs inoculated with $\mathrm{CF}$ gained similar flora to $\mathrm{CV}$ guinea-pigs flora, and we concluded that these LF guinea-pigs are available to be used as a source of intestinal flora for SPF colonies. The growth rate and the fertility of the SPF guinea-pigs were the same as those of the original Hartley guineapigs, and the animals were free from all pathogens tested. These results were satisfactory for a SPF guineapig breeding colony.

\section{References}

1. Boot, R., Angulo, A.F., and Walvoort, H.C. 1989. Clostridium difficile-associated typhlitis in specific pathogen free guinea-pigs in the absence of antimicrobial treatment. Lab. Animals 23: 203-207.

2. Boot, R., Koopman, J.P., Kruijt, B.C., Lammers, R.M., Kennis, H.M., Lankhorst, A., Welling, G.W., and Hectors, M.P.C. 1989. The 'normalization' of germ-free guinea-pigs with host-specific caecal microflora. Lab. Animals 23: 4852.

3. Boot, R. and Walvoort, H.C. 1986. Opportunistic infections 
in hysterctomy-derived, barrier-maintained guinea-pigs. Lab. Animals 20: 51-56.

4. Hazenberg, M.P. and Cluster-van Lieshout, L.M.C. 1976. Conversion of germ-free mice to the normal state by clostridia. Z. Versuchstienk. 18: 185-190.

5. Itoh, K. and Mitsuoka, T. 1980. Production of gnotobiotic mice with normal physiological functions. I. Selection of useful bacteria from feces of conventional mice. $Z$. Versuchstierk. 22: 173-178.

6. Itoh, K., Urano,T., and Mitsuoka,T. 1986. Colonization resistance against Pseudomonas aeruginosa in gnotobiotic mice. Lab. Animals 20: 197-201.

7. Itoh, K., Mitsuoka, T., Sudo, K., and Suzuki, K. 1983. Comparison of fecal flora of mice based upon different strains and different housing conditions. Z. Versuchstierk. 25: $135-146$.
8. Owen, D.G. and Porter, G. 1967. The establishment of a colony of specified-pathogen-free guinea-pigs. Lab. Animals 1: 151-156.

9. Van der Waaiji, D., Berghuis-de Vries, J.M., and Lekkerkerk-van der Wers, J.E.C. 1971. Colonization resistance of the digestive tract in conventional and antibiotic-treated mice. J. Hyg. 69: 405-411.

10. Yanabe, M., Shibuya, M., Gonda, T., Asai, H., Tanaka, T., Narita, T., Sudou, K., Matsui, T., and Itoh, K. 1999. Production of ex-germfree rabbits for establishment of specific pathogen-free colonies. Exp. Anim. 48: 79-86.

11. Yanabe, M., Shibuya, M., Gonda, Y., Asai, H., Tanaka, T., Narita, T., Sudo, K., and Itoh, K. 1999. Establishment of specific pathogen-free rabbit colonies with limited-flora rabbits associated with conventional rabbit flora, and monitoring of their cecal flora. Exp. Anim. 48: 101-106. 